\title{
Anthrovision
}

Vaneasa Online Journal

$2.1 \mid 2014$

Anthropologie et Numérique

\section{La force des médias pendant le « cyclone postélectoral » ivoirien}

\section{Véronique Duchesne}

\section{(2) OpenEdition}

1 Journals

\section{Édition électronique}

URL : http://journals.openedition.org/anthrovision/681

DOI : 10.4000/anthrovision.681

ISSN : 2198-6754

\section{Éditeur}

VANEASA - Visual Anthropology Network of European Association of Social Anthropologists

Référence électronique

Véronique Duchesne, «La force des médias pendant le « cyclone postélectoral » ivoirien », Anthrovision [En ligne], 2.1 | 2014, mis en ligne le 01 août 2013, consulté le 23 septembre 2019. URL : http:// journals.openedition.org/anthrovision/681; DOI : 10.4000/anthrovision.681

Ce document a été généré automatiquement le 23 septembre 2019

(c) Anthrovision 


\title{
La force des médias pendant le « cyclone postélectoral » ivoirien
}

\author{
Véronique Duchesne
}

1 La bataille médiatique qui a accompagné le conflit postélectoral ivoirien durant l'année 2011 n'a guère retenu l'attention des politologues et des spécialistes des média, encore moins celle des anthropologues. Pourtant on sait la force des médias comme vecteur de l'idéologie dans les batailles de pouvoir, et plus particulièrement dans les pays d'Afrique subsaharienne. La transmission idéologique est devenue quasi immédiate par le « contact médiatique » via la radio, la télévision et Internet notamment. J'ai choisi de présenter cinq "vignettes» sonores et audiovisuelles qui ont été diffusés par les médias ivoiriens et internationaux entre le 8 mars et le 17 juillet 2011. Ces " vignettes ", je les ai vues et entendues, en direct, en même temps que les compatriotes ivoiriens, dans le pays et dans la diaspora. Il s'agit, chronologiquement: 1/ des cris et lamentations d'Ivoiriennes lors de la Journée de la femme; 2/ des images de l'arrestation de Laurent Gbagbo; 3/ de l'allocution du Président du Conseil constitutionnel ; $4 / \mathrm{du}$ discours de la Grande Chancelière de l'Ordre National Ivoirien lors de l'investiture d'Alassane Ouattara; 5/ de la première harangue de Charles Blé Goudé en exil. Ces « vignettes » médiatiques suscitent un certain nombre de réflexions quant à l'instrumentalisation des média à des fins politiques et idéologiques en Côte d'Ivoire, et plus largement en Afrique subsaharienne. Elles visent également à montrer comment la radio, la télévision et Internet interagissent dans la transmission idéologique.

8 mars 2011 : La Journée internationale de la femme

SONS D'ARCHIVES

Sur les ondes de La Voix du Golf, à Abidjan, des femmes crient leur colère: elles y apostrophent les chefs religieux pour qu'ils « tranchent la vérité », elles y supplient la mère de Gbagbo de parler à son fils. 
Sur les ondes internationales d'Africa ${ }^{\circ} 1$, des femmes qui ont perdu leurs sœurs à Abobo font entendre leurs pleurs et clament : « On est fatigué ! » [« Il faut que nos souffrances cessent »].

2 A l'occasion de la Journée internationale de la femme, des Ivoiriennes soutenant Alassane Ouattara manifestent dans plusieurs communes d'Abidjan (Koumassi, Treichville, Attécoubé) pour dénoncer la mort de sept d'entre elles tuées par balles ${ }^{1}$ le 3 mars dans la commune d'Abobo, au nord d'Abidjan, alors qu'elles scandaient les slogans: "Gbagbo, dégage!", "On ne veut pas de Gbagbo", "Alassane président". Ce même jour, des organisations féminines de la société civile font des déclarations dans la presse : l' Organisation des Femmes Actives de Côte d'Ivoire (OFACI) et le Centre Féminin pour la démocratie et les droits humains en Côte d'Ivoire (CEFCI) notamment, pour rendre hommage à «[leurs] valeureuses martyres, tombées sur le champ de bataille de la démocratie ».

3 Alors qu'à Abidjan on peut entendre des femmes crier leur colère sur les ondes de $L a$ Voix du Golf, radio émettant depuis l'hôtel du Golf à Abidjan où Alassane Ouattara s'est réfugié, à Paris également, des membres des familles endeuillées d'Abobo déversent leur douleur et leur colère à l'antenne de la radio internationale Africa $n^{\circ} 1$. Quelques jours après, dans l'émission Le journal des éditeurs sur Africa $\mathrm{n}^{\circ} 1$, une ministre du gouvernement ( fantoche») de Laurent Gbagbo est interviewée par la journaliste Eugénie Diecky sur ce sujet et remet en question la réalité des faits, avançant que la mort des femmes d'Abobo ne serait qu'une mascarade. De même, les journalistes de la RTI (radio télévision ivoirienne) qui ont diffusé les images de la tuerie enregistrée par un téléphone portable ont d'abord affirmé qu'il s'agissait d'une mise en scène et que le sang visible sur les images n'était en fait qu'une boisson locale de couleur rouge (appelée bissap). De nombreuses photographies de femmes ensanglantées gisant sur le sol ainsi que des vidéo ont circulé sur Internet (sur les sites Abidjan.net et Youtube) et ont été qualifiées de " trucages » par les partisans du pouvoir en place. Il est intéressant de souligner que la manipulation des images est avancée par ceux qui ont détenu le monopole de l'information télévisuelle pendant toute la période pré-électorale puis pendant les premiers mois de la période postélectorale.

4 Alors ces femmes ont-elles été tuées ou s'agissait-il d'une «tromperie» via le numérique ? Une fois passée l'effervescence de l'actualité, beaucoup sont restés sur leur position gardant à l'esprit les discours qui ont accompagné la diffusion de ces images. Et lorsque finalement un accord sur la réalité des faits est établi, les versions diffèrent concernant les auteurs de la tuerie: la RTI (Radio-Télévision Ivoirienne) désigne comme auteurs de ce massacre les rebelles loyaux au premier ministre Guillaume Soro tandis que la TCI (Télévision de Côte d'Ivoire) accuse les forces de défense et de sécurité (FDS) loyales à Laurent Gbagbo.

5 Rappelons qu'après l'annonce du résultat des élections présidentielles depuis le Golf hôtel, les chaînes France 24 et TV5 ont été suspendues d'émettre. En janvier, la TCI (Télévision de Côte d'Ivoire) est créée par Ouatara et ses proches et émet depuis l'hôtel du Golf - elle est accessible sur Internet et localement grâce à un décodeur. Durant trois mois se déroule une véritable guerre entre les deux sources télévisuelles d'information, RTI et TCI, creusant encore l'écart idéologique entre les deux camps.

Début avril 2011, les équipements de la RTI sont détruits durant la bataille d'Abidjan et à partir du 16 avril la TCI emprunte le canal de l'ancienne RTI. 
11 avril 2011 : Arrestation de Laurent Gbagbo

\section{IMAGES D'ARCHIVES}

Le visage défait de Laurent Gbagbo passe en boucle sur les écrans du monde entier. Sans son synchrone, avec la voix du commentaire de la TCI.

7 Laurent Gbagbo a vécu les derniers jours avant son arrestation, réfugié dans le sous-sol de la résidence présidentielle, entouré de membres de sa famille proche (sa mère, son épouse, son fils, sa fille et ses petits enfants), de plusieurs généraux, de nombreux conseillers $^{2}$ et de plusieurs pasteurs évangéliques ${ }^{3}$. Ceux qui étaient présents ont décrit une ambiance quasi mystique, avec Simone Gbagbo constamment recueillie dans la prière. Le réalisateur ivoirien Sidiki Bakaba ${ }^{4}$ avait également sa caméra, soucieux d'immortaliser ce dernier épisode de résistance.

8 La vidéo de l'arrestation de Laurent Gbagbo, en maillot de corps, les yeux hagards, a été diffusée par la TCI puis repris en boucle sur les écrans du monde entier : Laurent Gbagbo était en train de parler sans que l'on entende le son de sa voix. Cette image d'un homme ordinaire et sans voix, concourt à le rendre pathétique mais nullement à faire de lui un vaincu. La dimension religieuse de l'attachement de ses partisans à Laurent Gbagbo ne doit pas être négligée. "C'est Dieu qui l'a mis là » est la formule consacrée pour tous ceux qui, comme Laurent et Simone Gbagbo, évangéliques convertis, le considèrent investi d'une mission divine pour sauver la Côte d'Ivoire. La vidéo diffusée tend plutôt à le transformer en une icône ${ }^{5}$.

6 mai 2011 : Discours du président du Conseil constitutionnel lors de la prestation de serment d'Alassane Ouatara.

\section{IMAGES D'ARCHIVES}

Palais présidentiel. Le Président du Conseil Constitutionnel, Paul Yao N’Dré, de retour de son exil au Ghana, va faire prêter serment au nouveau président de la République Alassane Ouattara. Il lâche à la fin de son allocution devant des millions de téléspectateurs : « Nous sommes allés tous trop loin, me disait un ami du RDR. Et Monsieur le Président, il précisait, nous n`avons pas tous voulu entendre raison, Satan nous a possédés tous ! (Diffusé en direct par la TCI, Télévision de Côte d'Ivoire).

Dans sa déclaration télévisée, le président du Conseil constitutionnel invoque Satan pour expliquer le débordement de violence qui a enflammé Abidjan et la Côte d'Ivoire. Cette phrase fut reprise dans la presse puis par nombre d'Ivoiriens qui se dirent choqués : comment un homme pouvait-il publiquement se dédouaner de sa propre responsabilité en faisant référence à Satan ? Il faut sans doute rappeler que jusqu'à la destruction des équipements de la RTI, le présentateur du journal télévisé finissait toujours par la formule: « Que Dieu bénisse la Côte d'Ivoire ».

21 mai 2011 : Discours de la Grande Chancelière de l'Ordre National Ivoirien lors de l'investiture d'Alassane Ouattara. 


\section{IMAGES D'ARCHIVES}

A Yamoussoukro, la ville d'Houphouët Boigny, devant soixante délégations étrangères, vingt et un chef d'Etats et toutes les élites de la Côte d'Ivoire, a lieu la cérémonie d'investiture du Président Alassane Ouattara : Henriette Diabaté, première femme Grande Chancelière de Côte d'Ivoire, lui remet solennellement la grande Croix (diffusé en direct par la TCI, Télévision de Côte d'Ivoire).

Ce 21 mai, sont réunis au-devant de la scène deux militants de longue date du RDR (Rassemblement des Républicains) : Alassane Ouattara qui dirigeait le parti jusqu'à son élection et Henriette Diabaté qui en était la secrétaire générale depuis 1999. Celle-ci nommée Grande Chancelière de l'Ordre National Ivoirien par le président Alassane Ouattara apparaît très émue lors de son discours. Sur Dailymotion, un extrait de 9 secondes donne à entendre : "Vous-même monsieur le ...préfet", puis elle rectifie aussitôt «monsieur le président ». Le titre donné à la vidéo est : " Ouattara préfet de Sarkozy ». Le lapsus de l'oratrice quasiment inaudible en direct, permet aux adversaires de Ouattara, grâce à un usage différé de l'image, de tirer parti de son émotion ${ }^{6}$. L'usage détourné des images à des fins politiques et idéologiques est très largement pratiqué sur Internet mais n'a pas fait l'objet d'études spécifiques.

17 juillet 2011: Première harangue de Charles Blé Goudé en exil

\section{SON D'ARCHIVES}

« [...]Tous ceux qui ont participé à la campagne de Gbagbo Laurent sont traqués, sont poursuivis, sont tués, leurs comptes sont bloqués [...].

Vous avez un président qui est du nord, un premier ministre qui est du nord, un ministre de l'intérieur qui est du nord, un ministre de l'industrie du nord, un ministre de l'agriculture du nord, le secrétaire général du gouvernement du nord, le directeur administratif et financier de la république du nord, le président $\mathrm{du}$ port d'Abidjan du nord, du port de San Pedro du nord, la plupart des ambassadeurs sont du nord. Il n'y a que de l'intelligence du Nord. Les autres ethnies de la Côte d'Ivoire n'ont aucun cadre, n'ont aucune intelligence? [...]

En fait, on me poursuit, on m'en veut, pas parce que je mobilise, on m'en veut pour la cause pour laquelle je mobilise. On m'en veut aussi pour mon ethnie puisque tous ceux qui sont de l'ouest, du centre ouest, du sud de la Côte d'Ivoire, de l'est de la Côte d'Ivoire aujourd'hui sont des persona non grata en Côte d'Ivoire. Je dénonce cela, cet élan tribal [...]»

(Diffusé sur Internet puis extraits sonores diffusés sur RFI, Radio France Internationale, dès le lendemain matin).

Depuis l'extérieur du pays, Charles Blé Goudé, le " général » des Jeunes Patriotes, figure de proue du régime de Laurent Gbagbo contre qui un mandat d'arrêt international a été délivré, réussit à se faire entendre par le biais de Dailymotion puis de RFI (Radio France Internationale). Dans sa harangue, Blé Goudé joue sur la peur que partagent nombre de ceux qui ont choisi l'exil, s'estimant en danger dans leur pays. Il continue d'instiller la haine à l'encontre de ceux «du nord» et veut réduire le conflit à une lutte entre 
groupes ethniques ${ }^{7}$. Ceux qui ont fui, pour la plupart dans un pays voisin de la sousrégion (Ghana, Togo, Bénin) ont pu continuer à alimenter la peur et la haine depuis l'extérieur du pays grâce à leurs connections avec des médias internationaux, tels que RFI dans l'exemple cité.

Force est de rappeler que les médias ont joué un rôle prépondérant dans l'aggravation de la crise socio-politique. Dans un pays où la majorité de la population lit très peu la presse et se tient informée essentiellement par la radio et la télévision, la RTI, puis la TCI, ont pu servir d'arme de conviction massive à l'intérieur du pays mais également au sein de la diaspora par le biais d'Internet. Une étude des archives télévisuelles, si elles n'ont pas été entièrement détruites, pourra montrer comment la RTI fut, avant et après les élections, un véritable outil de propagande diffusant des messages de haine. La radio, la télévision et Internet représentent une force indispensable pour ceux qui aspirent au pouvoir, en Côte d'Ivoire comme ailleurs en Afrique. Souhaitons que des anthropologues ${ }^{8}$, politologues ${ }^{9}$ ou historiens se penchent plus avant sur ce que l'on appelle fort justement le « quatrième pouvoir ". d'Abidjan émet à nouveau, reformatée sous le nom de RTI2.

Sigle des radios et télévisions ivoiriennes, avant avril 2011 et après avril 2011

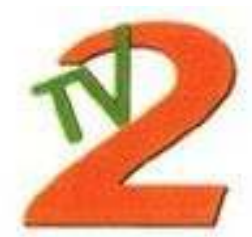

(c) Droits réservés

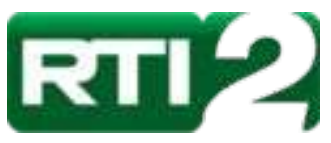

(C) DROITS RÉSERVÉS

Le mercredi 17 juillet 2013, la Haute Autorité de la Communication Audiovisuelle (HACA) a animé une session d'information, sur le thème : «Régulation et libéralisation du secteur de l'Audiovisuel : enjeux et intérêts pour nos populations». Il s'agira pour l'Etat de Côte d'Ivoire à travers la HACA de permettre à des entités privées de disposer de chânes de télévisions, d'ici juin 2015.

17 A suivre ...de près. 


\section{NOTES}

1. Comme dans beaucoup de guerres contemporaines, les civils ont été les principales victimes car il faut bien parler de "guerre»: la victoire a finalement été celle des armes, au grand désespoir de tous les Ivoiriens qui se sont présentés si nombreux aux urnes. Cette participation active des femmes n'a guère été relayée par les média français et internationaux

2. Damana Pickas, ancien membre de la CEI, Commission électorale indépendante - qui a notamment, devant les yeux du monde entier rivés sur leurs écrans de télévision, arraché et déchiré la feuille que Bamba Yacouba s'apprêtait à lire pour la proclamation des résultats du second tour des élections qui donnait Alassane Ouattara vainqueur - est resté dans la résidence présidentielle aux côtés de Laurent Gbagbo jusqu'à la veille de son arrestation puis est parti en exil.

3. Rappelons qu'après son élection à la présidence en octobre 2000, Laurent Gbagbo, pour conforter sa fragile légitimité, décidait de s'appuyer sur les Ivoiriens chrétiens, majoritaires dans le Sud. A partir de 2002, le conflit est présenté comme étant le combat des forces du bien incarnées par le régime en place, contre les forces du mal, les rebelles considérés comme majoritairement musulmans. Dans la continuité de cette idéologie, la résistance des partisans de Laurent Gbagbo après le second tour des élections présidentielles est pour eux assimilée à un combat spirituel, l'impérialisme français et plus largement occidental n'étant qu'une figure du mal radical dont les partisans d'Alassane Ouattara, qui plus est vus comme des musulmans, sont l'incarnation. Cf. S. Raynal, «En Côte d'Ivoire, les forces du bien contre les forces du mal», Hérodote, n¹19, 2005. D'ailleurs si la hiérarchie catholique de Côte d'Ivoire a pris publiquement parti pour Laurent Gbagbo, c'est très probablement par crainte d'être gouvernée par des musulmans.

4. Sidiki Bakaba fut blessé lors de la prise de la résidence et fut transféré en France pour y être soigné.

5. La phrase postée par un internaute (le 21 avril 2011) est à cet égard tout à fait significative: «De toute façon [qu'il soit] mort ou vivant; libre ou en prison la légende Gbagbo continuera!» Sur le blog «Côte d'Ivoire- Appel au combat de Marie Antoinette Singleton », publié par Allain Jules le 18 avril 2011.

6. La page du journal Le Temps (pro Gbagbo) du 23 mai 2011 relatant sur un ton sarcastique ce micro-événement est lisible sur le site Cameroon Web News.

7. Blé Goudé dans sa tirade omet de citer au sein du nouveau gouvernement: Anne Ouloto, ministre de la Salubrité urbaine et militante de longue date du Rassemblement des femmes républicaines (RFR), et Dagobert Banzio, ministre du commerce, tous deux originaires de l'ouest du pays, région dont la population a beaucoup souffert lors du conflit armé.

8. Cf. Abu-Lughod, L, Ginsburg, F. et Larkin, B. (ed.), Media Worlds: Anthropology on New Terrain, Berkeley, University of California Press, 2002 et Abu-Lughod, L, Dramas of Nationhood: The Politics of Television in Egypt, Chicago, University of Chicago Press. Abu-Lughod part du constat de la pauvreté ethnographique des recherches sur les médias, qui s'en tiennent souvent à des considérations générales sur la fabrication ou la consommation des produits culturels.

9. A l'instar d'Émile A. Tozzo dans son article « La réforme des médias publics en Afrique de l'Ouest », Politique africaine 1/2005 ( ${ }^{\circ}$ 97), p. 99-115, dans lequel il écrit au sujet de la RTI : «Par un décret présidentiel du 24 décembre 2004, la Radio-Télévision ivoirienne (RTI) est passée du statut de "société anonyme" à celui de "société d'État». Ce changement de statut est le quatrième que connaît la RTI en moins de seize mois, après quelques valses entre celui de "société d'économie mixte de type particulier» et de "société anonyme». En dépit des circonstances particulières de cette mutation, dans un contexte de conflit armé où la reprise en 
main des armes de guerre audiovisuelles est inévitable, cette valse de l'assise juridique et administrative de la radio-télévision publique ivoirienne est symptomatique du piétinement de la réforme des médias publics en Afrique de l'Ouest et révélatrice de la lutte politique persistante pour le contrôle de l'information ».

\section{RÉSUMÉS}

La bataille médiatique qui a accompagné le conflit postélectoral ivoirien durant l'année 2011 n'a guère retenu l'attention des politologues et des spécialistes des média, encore moins celle des anthropologues. Pourtant on sait la force des médias comme vecteur de l'idéologie dans les batailles de pouvoir, et plus particulièrement dans les pays d'Afrique subsaharienne. Cinq «vignettes» sonores et audiovisuelles qui ont été diffusées par les médias ivoiriens et internationaux entre le 8 mars et le 17 juillet 2011 sont présentées et sont le support de réflexions quant à l'instrumentalisation des média à des fins politiques et idéologiques en Côte d'Ivoire, et plus largement en Afrique subsaharienne. Elles visent également à montrer comment la radio, la télévision et Internet interagissent dans la transmission idéologique.

The media battle, which occurred in 2011 during the post electoral conflict in Ivory Coast didn't catch the attention of political scientist, media specialist and even less anthropologists. However, we know quite well the important role of media as ideological means during political power confrontation especially in African sub Saharan countries. Five shorts teaser which were broadcast by Ivorian and international media between the $8^{\text {th }}$ of March and the $17^{\text {th }}$ of July 2011 are analysed as means of reflexions to understand the media instrumentalisation for political and ideological purposes specially in Ivory Coast and more broadly in Sub-Saharan Africa. This contribution offers an insight on the ways Radio, Television, and Internet interact with the ideological transmission.

La batalla digital que acompañó al conflicto postelectoral en Costa de Marfil durante el 2011 no ha despertado el interés de politólogos y especialistas de los media, y aún menos el de los antropólogos. Sin embargo, sabemos de la fuerza de los media como vector ideológico en las guerras de poder, y especialmente en el África Subsahariana. Estel artículo presenta cinco fragmentos sonoros y audiovisuales difundidos tanto por los media de Costa de Marfil como por los media internacionales entre el 8 de marzo y el 17 de julio de 2011. En base a estos fragmentos se construye una reflexión sobre la instrumentalización de los media con fines políticos e ideológicos en Costa de Marfil y, en general, en toda el África Subsahariana. Así, se pretende igualmente mostrar cómo la radio, la televisión e Internet intervienen en la transmisión ideológica.

\section{INDEX}

Mots-clés : Côte d'Ivoire, bataille médiatique, idéologie, anthropologie visuelle

Palabras claves : Costa de Marfil, guerra Mediática, ideología, antropología visual

Keywords : Ivroy Coast, media battle, ideology, visual anthropology 
AUTEUR

VÉRONIQUE DUCHESNE

Anthropologue et vidéaste, maître de conférences à l'Université Paris Descartes 